\title{
DETERMINAĈ̃O DA VARIABILIDADE EM ISOLADOS DE Colletotrichum lindemuthianum POR MEIO DE MARCADORES MORFOLÓGICOS E CULTURAIS
}

\author{
Determination of variability in isolates of Colletotrichum lindemuthianum based \\ on morphological and cultural markers
}

\author{
Breno Oliveira de Souza ${ }^{1}$, Elaine Aparecida de Souza ${ }^{2}$, Maria Cristina Mendes-Costa ${ }^{3}$
}

\begin{abstract}
RESUMO
Colletotrichum lindemuthianum (teleomorfo Glomerella cingulata f. sp. phaseoli) apresenta ampla variabilidade genética, demonstrada por suas características morfológicas. Com este trabalho, objetivou-se caracterizar, por meio de marcadores morfológicos, diferentes isolados de $C$. lindemuthianum e identificar marcadores morfológicos com uso potencial em análises genéticas. Foram avaliados os seguintes caracteres morfológicos e culturais: cor e textura das colônias, compatibilidade vegetativa e sexual, índice de velocidade de crescimento micelial (IVCM), diâmetro colonial (DC), capacidade de esporulação (CE), dimensões e formas conidiais, dimensões dos ascósporos, formação de estruturas reprodutivas e formação de anastomoses entre hifas e conídios. Os resultados obtidos demonstraram que os isolados de $C$. lindemuthianum possuem ampla variabilidade genética para todas as características avaliadas e que a forma do conídio pode ser usada como marcador morfológico em análises genéticas.
\end{abstract}

Termos para indexação: Caracteres morfológicos e culturais, dimensões e forma do conídio, anastomoses entre conídios e hifas, compatibilidades vegetativa e sexual.

\section{ABSTRACT}

Colletotrichum lindemuthianum (teleomorfo Glomerella cingulata f. sp.phaseoli) presents wide genetic variability, demonstrated by its morphological traits. The objective of this study was to characterize morphological markers in different isolates of $C$. lindemuthianum and, to identify useful morphological markers in genetic analyses. The following morphological and cultural traits were evaluated: color and texture of the colonies, vegetative and sexual compatibility, micelial growth index (MGI), colonial diameter (CD), esporulation capacity (EC), conidia dimensions and form, ascospores dimensions and formation of reproductive structures. The data showed wide genetic variability for all traits and that conidial form can be used as morphological marker in genetic analysis.

Index terms: Morphological and cultural traits, conidia dimensions and form, conidia and hifas anastomosis, vegetative and sexual compatibility.

(Recebido em 20 de outubro de 2005 e aprovado em 7 de novembro de 2006)

\section{INTRODUÇÃO}

Colletotrichum lindemuthianum (Sacc. \& Magn.) Scrib. (teleomorfo Glomerella cingulata (Stonem) Spauld \& Schrank f. sp. phaseoli Kimati) agente causal da antracnose do feijoeiro (Phaseolus vulgaris L.) apresenta ampla variabilidade genética, demonstrada pela ocorrência de vários patótipos, características morfológicas e culturais. Vários mecanismos são responsáveis pela variabilidade genética em fungos. Muitos destes fenômenos importantes observados em patógenos de plantas ainda não foram explicados adequadamente.

C. lindemuthianum apresenta micélio apocítico e ramificado e se reproduz de forma assexual. Os conídios formam massa de cor salmão ou mel, são hialinos, unicelulares, oblongos e cilíndricos, apresentando as extremidades arredondadas (Sutton, 1992).
A fase sexuada Glomerella cingulata f. sp. phaseoli, até o presente momento, nunca foi encontrada na natureza. Kimati \& Galli (1970) foram os primeiros a observarem a forma sexuada em laboratório por indução, no entanto, esta pode ser obtida facilmente pelo seu desenvolvimento espontâneo em cultivos de laboratório (Camargo Júnior, 2004; Mendes-Costa \& Souza, 2005). Estudos genéticos e citológicos foram realizados em $G$. cingulata até a década de 1950, entretanto após este período não se encontram relatos na literatura até o trabalho de Kimati \& Galli (1970). Relatos posteriores de Batista \& Chaves (1982), Bryson et al. (1992) e Mendes-Costa (1996) revelam que a utilização da forma meiospórica para estudos genéticos tem sido pouco explorada. Mais recentemente, Roca et al. (2003) descreveram as etapas do desenvolvimento de peritécios de G. cingulata f. sp.

1Doutorando - Departamento de Fitopatologia/DFP - Universidade Federal de Lavras/UFLA - Cx. P. 3037 - $37200-000$ - Lavras, MG souzabio@yahoo.com.br

²Doutora, Professora - Departamento de Biologia/DBI - Universidade Federal de Lavras/UFLA - Cx. P. 3037-37200-000 - Lavras, MG - easouza@ufla.br ${ }^{3}$ Doutora, Professora - Departamento de Biologia - Centro Universitário de Lavras/UNILAVRAS - Rua Padre José Poggel, 506, Centenário - Lavras, MG mcmcosta@unilavras.edu.br 
phaseoli. Ishikawa \& Souza (2004) fizeram um estudo morfológico e Mendes-Costa \& Souza (2005) identificaram os genótipos correspondentes a características culturais.

Devido à ampla variabilidade genética existente, $\mathrm{o}$ estudo das características morfológicas em fitopatógenos é importante para serem utilizadas como marcadores morfológicos em estudos genéticos. Portanto, o presente trabalho objetivou estudar a variabilidade de $C$. lindemuthianum por meio de marcadores morfológicos, assim como, identificar marcadores morfológicos com uso potencial em análises genéticas.

\section{MATERIAL E MÉTODOS}

Todos os procedimentos foram realizados no Departamento de Biologia da Universidade Federal de Lavras (UFLA) e no Centro Universitário de Lavras (UNILAVRAS).

Os isolados LV4, patótipo 81 (Viçosa-MG), LV33, patótipo 337 (Viçosa-MG), LV47, patótipo 593 (Cristalina - GO), LV51, patótipo 73 (Lavras-MG), LV71, patótipo 65 (Monte Carmelo-MG), LV76, patótipo 2047 (Costa Rica) e LV77, patótipo 89 (Lavras-MG) são da forma assexual e os isolados LV79, LV80, LV81, LV82, LV83 e LV84 (DBI/UFLA) são da forma sexual. Os isolados foram obtidos por isolamento, com exceção de LV83 e LV84 que foram reisolados utilizando-se procedimentos adaptados de Mendes-Costa (1996).

Para verificar a compatibilidade vegetativa e sexual entre os isolados estes foram confrontados dois a dois segundo a metodologia descrita por Bryson (1990) e Rodriguez-Guerra et al. (1999). Um setor branco que surgiu na colônia do isolado LV51 foi confrontado com os demais isolados utilizados no estudo.

Foram avaliados os seguintes caracteres morfológicos:

\section{Índice de velocidade de crescimento micelial (IVCM):}

O IVCM foi medido segundo Nechet (1999) e estimado utilizando-se a fórmula apresentada por Oliveira (1991):

$$
\operatorname{IVCM}=(\mathrm{D}-\mathrm{Da}) / \mathrm{N}
$$

Sendo:

IVCM=índice de velocidade de crescimento micelial. $\mathrm{D}=$ diâmetro médio atual da colônia.

Da=diâmetro médio da colônia do dia anterior.

$\mathrm{N}=$ número de dias após a inoculação.

\section{Diâmetro da colônia:}

O diâmetro das colônias de $C$. lindemuthianum foi medido em centímetros, por um período de 15 dias. Os isolados foram cultivados em meio de cultivo $\mathrm{M}_{3}$ (Junqueira et al. 1984). Foram avaliadas cinco placas por isolado.

\section{Capacidade de esporulação:}

Foram obtidas suspensões conidiais a partir da esporulação em vagem de feijão esterilizada mantida em tubos de ensaio, utilizando-se $2 \mathrm{~mL}$ de água por tubo. Posteriormente, procedeu-se como descrito por Dias (2002).

\section{Cor e textura das colônias:}

Os isolados crescidos em BOD (fotoperíodo 12h) a $21^{\circ} \mathrm{C}$ sobre meio $\mathrm{M}_{3}$ sólido (Junqueira et al., 1984) foram observados com sete, quinze e trinta dias de idade. Foi utilizada a escala de cores Munsell ${ }^{\circledR}$ Soil Color Charts (1979).

\section{Presença de estruturas reprodutivas:}

Após sete dias de crescimento dos isolados em lâminas de microcultivo, foi observada a presença de estruturas reprodutivas.

\section{Dimensão dos esporos:}

Foram medidos 30 conídios e 30 ascósporos em cada amostra. A relação comprimento/largura e a forma dos conídios foram descritas segundo Feitosa et al. (1977). Estes autores consideram $\mathrm{Ccc}=$ cilíndrico muito curto, relação comprimento/largura menor que 2,00; $\mathrm{Cc}=$ cilíndrico curto, relação entre 2,00 e 2,50; $\mathrm{C}=$ cilíndrico, entre 2,50 e 3,$00 ; \mathrm{f}=$ fusiforme, entre 3,00 e 3,$50 ; \mathrm{ff}=$ fusiforme muito alongado, relação maior que 3,5 .

As avaliações citológicas foram realizadas segundo Roca et al. (2003). Os conídios e os ascósporos foram medidos por meio de uma ocular OSM, acoplada ao microscópio de contraste de fase Luca DMLS na objetiva de 40x.

Os dados obtidos do índice de velocidade de crescimento micelial (IVCM), diâmetro da colônia, capacidade de esporulação, comprimento e largura dos conídios e ascósporos foram analisados em DIC (delineamento inteiramente casualizado). $\mathrm{O}$ crescimento micelial foi avaliado em DIC com parcelas subdivididas no tempo. Foi realizada uma análise de regressão do crescimento das colônias em função do tempo em dias. As análises estatísticas foram realizadas no programa SISVAR. 


\section{RESULTADOS E DISCUSSÃO}

Todas as características morfológicas avaliadas apresentaram ampla variabilidade genética. Entretanto, todas as comparações dos isolados dois a dois mostraramse incompatíveis (Figuras 1a e 1b), incluindo aqueles que envolveram o setor branco da linhagem LV51. Não foi observada a compatibilidade sexual entre os isolados avaliados (Figura 1c e 1d).

O estudo da compatibilidade vegetativa entre isolados é de extrema importância em estudos genéticos. Roca et al. (2003), avaliando diferentes isolados de $C$. lindemuthianum, verificaram a ocorrência de incompatibilidade vegetativa por meio do crescimento helicoidal destes isolados. Já, Rodriguez-Guerra et al. (1999) mostraram a formação de peritécios na linha de comparação. Muitos isolados confrontados produziram estruturas arredondadas estéreis denominadas prototécios. Relatos na literatura citam que, normalmente, cruzamentos compatíveis envolvem isolados de colônias claras com isolados de colônias escuras (Lucas, 1946; Mendes-Costa \& Souza, 2005).

Para o índice de velocidade de crescimento micelial (IVCM), apenas os isolados LV77 e LV76 diferiram estatisticamente dos demais pelo teste de Scott-Knott (Tabela 1). Na avaliação do crescimento micelial a interação isolados $\mathrm{x}$ tempo foi significativa $(\mathrm{P}<0,01)$, indicando que o crescimento dos isolados não foi coincidente ao longo dos dias. Os isolados apresentaram uma relação linear entre o crescimento da colônia e o tempo nos 15 dias de avaliação, sendo que o isolado LV47 foi o de crescimento mais lento e LV76 de crescimento rápido (Figura 2). Foi relatado por Feitosa et al. (1977) e Dias (2002) que diferentes isolados apresentavam diferenças significativas na velocidade de crescimento micelial em meio de cultura.

Em relação à capacidade de esporulação, os isolados apresentaram variação, destacando-se o isolado LV47 que apresentou esporulação significativamente superior aos demais (Tabela 1).

Todas as colônias de $C$. lindemuthianum apresentaram coloração variando de branco a cinza-claro (Figura $1 \mathrm{~g}$ e $1 \mathrm{~h}$ ) e, com o passar do tempo de cultivo, de cinza-escuro a negra (Figura 1i e 1j) com superfícies delicadamente aveludadas. Os isolados LV51 e LV33 formaram setores mais claros e de superfícies aveludadas, (Figura 1k) enquanto, as colônias dos isolados LV76 e LV77 foram as mais estáveis e as únicas que produziram a forma teleomórfica, produzindo peritécios (Figura 1f) inicialmente em aglomerados (Figura11). Com relação às características culturais, as cores das colônias variaram dentro das tonalidades de cores descritas para o gênero Colletotrichum (Sutton, 1992). Alguns acérvulos apresentaram setas enquanto outros eram desprovidos deste tipo de estrutura.

Os isolados LV33 e LV47 diferiram significativamente dos demais apresentando as menores dimensões quanto ao comprimento dos conídios (Tabela 2). Em relação à largura, o isolado LV51 apresentou os conídios mais largos $(6,48 \mu \mathrm{m})$ e o isolado LV47 os mais estreitos $(4,14 \mu \mathrm{m})$.

Apenas os isolados LV71 e LV51 apresentaram a forma dos conídios diferente dos demais (Tabela 2). De acordo com a relação comprimento/largura foram cilíndricos (Figura 1m), cilíndricos curtos (Figura 1n) e fusiformes (Figura1o) com as extremidades arredondadas. O tamanho médio dos conídios dos isolados foi próximo ao descrito por Rava \& Sartorato (1994) e Roca et al. (2003), no entanto, foi superior aos citados por Sutton (1992).

Com relação aos caracteres avaliados da forma teleomórfica houve grande variação nas médias para o tamanho dos ascósporos. Dos seis isolados avaliados cinco apresentaram diferenças estatisticamente significativas (Tabela 3). Roca et al. (2003), avaliando as médias do comprimento e largura dos ascósporos de três isolados de G. cingulata f. sp. phaseoli, verificaram que o isolado 531, que pertence ao patótipo 73, apresentou as maiores dimensões $(21,32 \times 7,17 \mu \mathrm{m})$ e o isolado 1013 (patótipo 1) as menores (18,26 x 4,94 $\mu \mathrm{m})$. Já Castro et al. (2005) observaram dimorfismo sexual nos ascósporos sendo os de tamanho normal com 21,7 mm de comprimento e os ascósporos anões com 15,1 $\mu \mathrm{m}$.

A avaliação do comprimento e largura dos conídios e ascósporos pode ser de extrema importância, pois isolados bem contrastantes em relação ao tamanho e forma dos mesmos podem ser muito úteis como marcadores morfológicos em análises genéticas. 


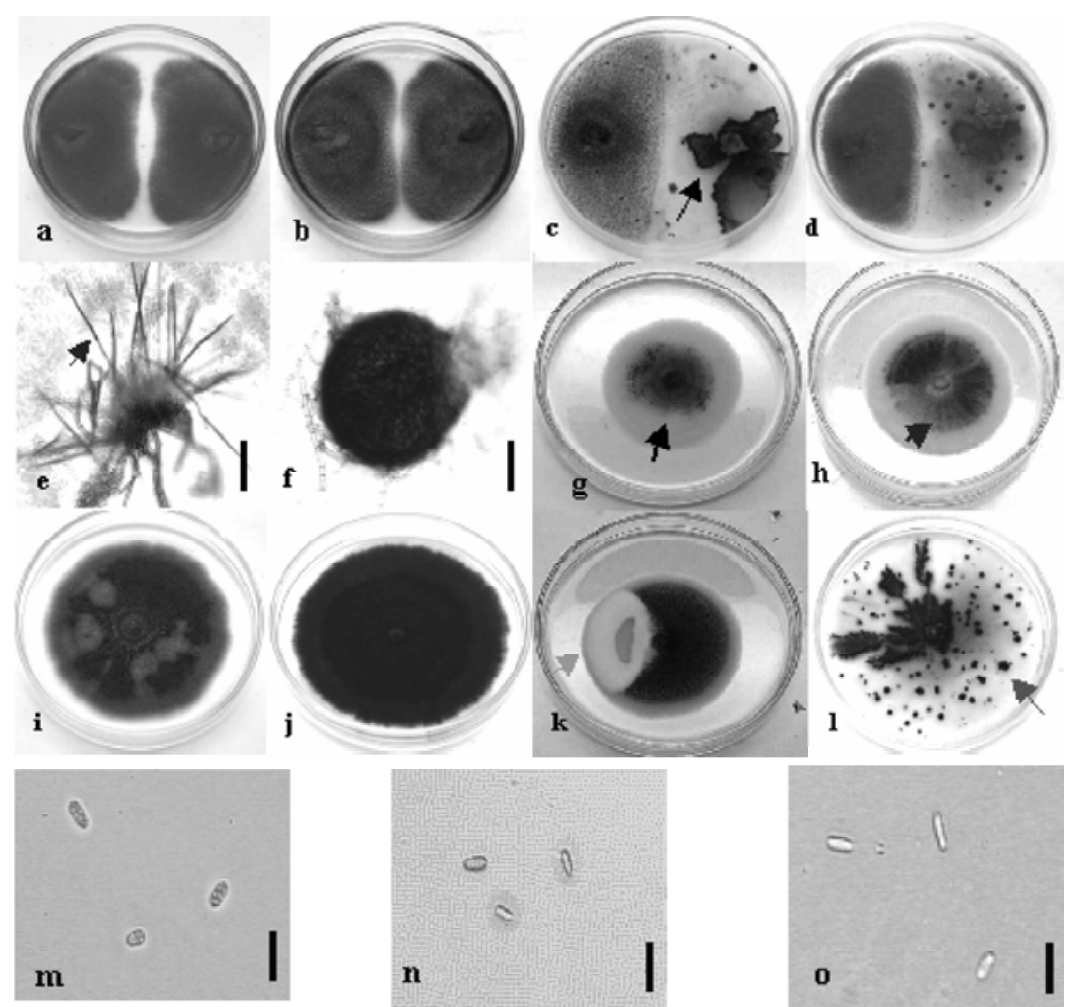

FIGURA 1 - Sequiência de imagens de colônias cultivadas em placas de Petri, de estruturas de reprodução e conídios de Colletotrichum lindemuthianum. Incompatibilidade vegetativa entre isolados de $C$. lindemuthianum; a - comparação entre colônias do patótipo 2047 e b - comparação entre colônias dos patótipos 65 e 2047; Incompatibilidade sexual entre isolados de Glomerella cingulataf. sp. phaseoli; c - LV79 confrontado com LV84 e d - LV80 com LV83. Linha de comparação formada no limite de setor dentro de um mesmo isolado (seta); Estruturas reprodutivas, esporodóquios (acérvulos) com a presença de seta (seta) - e, e peritécio estourado - f (Barras: $10 \mu \mathrm{m})$; Colônias de $C$. lindemuthianum aos sete dias de idade com coloração de branca (seta) - g, a cinza-claro (seta) - h, e aos trinta dias de idade com coloração de cinza-escuro-i, a negra - j; g - LV33, h-LV76, i - LV47 e j - LV71; Colônia do isolado do patótipo 73 -k, de $C$. lindemuthianum que formou setor mais claro e de superfície aveludada (seta) e colônia de G. cingulata f. sp. phaseoli-1, mostrando peritécios em aglomerado (seta); Formas dos conídios de C. lindemuthianum; m - cilíndricos (LV71), n - cilíndricos curtos (LV51), o - fusiformes (LV77) (Barras: $50 \mu \mathrm{m}$ ).

TABELA 1 - Índice de velocidade de crescimento micelial $(\mathrm{cm} /$ dia $)$ e capacidade de esporulação dos isolados de Colletotrichum lindemuthianum.

\begin{tabular}{lcc}
\hline Isolado & IVCM & Conídios $\mathbf{~ 1 0}^{\mathbf{6}}$ \\
\hline LV71 & $0,90 \mathrm{~b}^{1}$ & $15,58 \mathrm{c}^{1}$ \\
LV51 & $1,03 \mathrm{~b}$ & $5,52 \mathrm{~d}$ \\
LV4 & $0,93 \mathrm{~b}$ & $15,49 \mathrm{c}$ \\
LV77 & $1,07 \mathrm{a}$ & $21,96 \mathrm{~b}$ \\
LV33 & $0,98 \mathrm{~b}$ & $24,10 \mathrm{~b}$ \\
LV47 & $0,88 \mathrm{~b}$ & $50,92 \mathrm{a}$ \\
LV76 & $1,11 \mathrm{a}$ & $7,44 \mathrm{~d}$ \\
\hline
\end{tabular}

${ }^{1}$ Médias seguidas da mesma letra não diferem estatisticamente, pelo teste de Scott-Knott, a 5\% de probabilidade. 
LV71

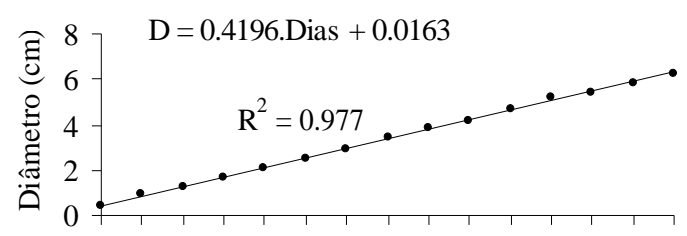

$\begin{array}{lllllllllllllll}1 & 2 & 3 & 4 & 5 & 6 & 7 & 8 & 9 & 10 & 11 & 12 & 13 & 14 & 15\end{array}$

Dias

\section{LV51}

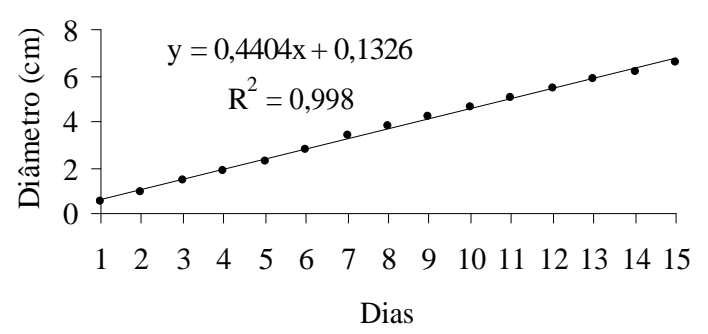

LV4

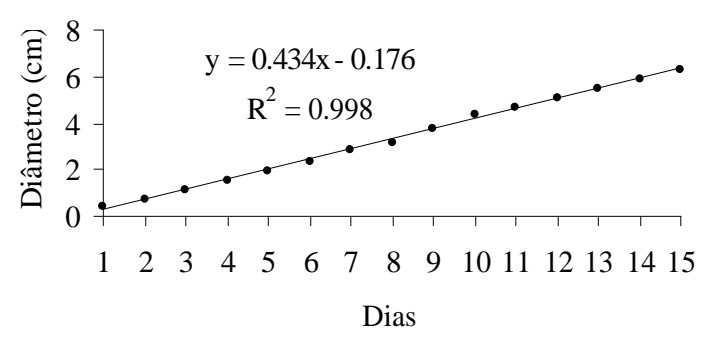

LV33

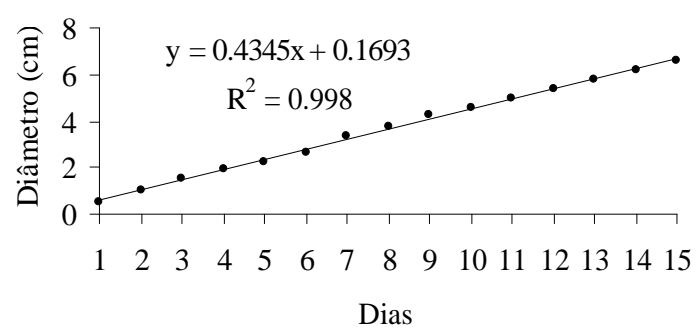

LV47

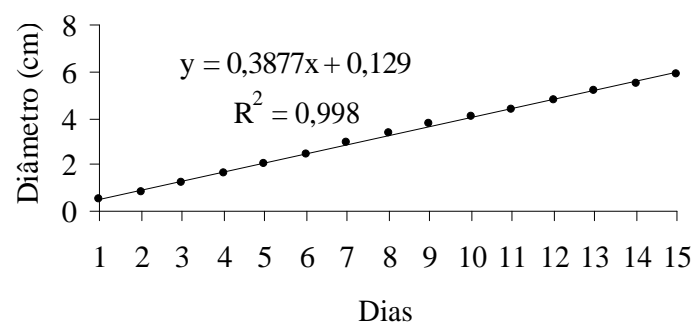

LV76

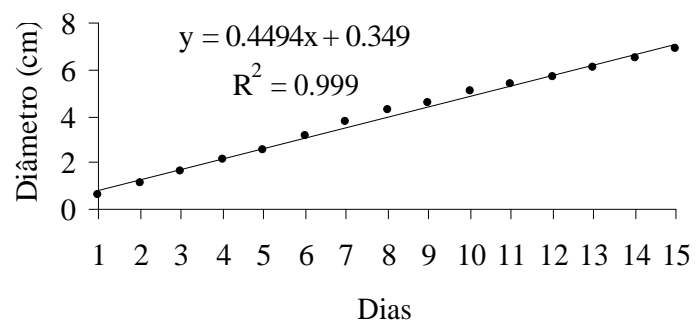

LV77

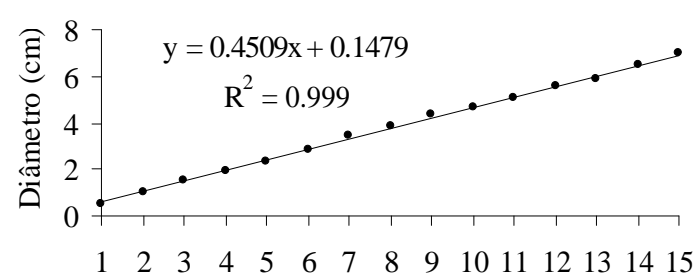

FIGURA 2 - Equação de regressão para o crescimento micelial de isolados de C. lindemuthianum. 
TABELA 2 - Dimensões $(\mu \mathrm{m})$ e forma dos conídios dos isolados de Colletotrichum lindemuthianum.

\begin{tabular}{|c|c|c|c|c|}
\hline \multirow[t]{2}{*}{ Isolados } & \multicolumn{2}{|c|}{$\begin{array}{l}\text { Médias e desvios padrão das dimensões dos } \\
\text { conídios }\end{array}$} & \multirow{2}{*}{$\begin{array}{c}\text { Relação } \\
\text { Comp./Larg. }\end{array}$} & \multirow{2}{*}{$\begin{array}{c}\text { Forma dos } \\
\text { conídios }\end{array}$} \\
\hline & Comprimento & Largura & & \\
\hline LV71 & $16,32 \pm 1,47 \mathrm{a}^{1}$ & $6,12 \pm 2,47 b^{1}$ & 2,66 & $* \mathrm{C}$ \\
\hline LV51 & $15,86 \pm 1,42 \mathrm{a}$ & $6,48 \pm 2,55 a$ & 2,45 & $* \mathrm{Cc}$ \\
\hline LV4 & $16,16 \pm 1,87 \mathrm{a}$ & $4,59 \pm 2,14 d$ & 3,45 & $* \mathrm{~F}$ \\
\hline LV77 & $15,74 \pm 1,29 a$ & $4,67 \pm 2,17 d$ & 3,36 & $* \mathrm{~F}$ \\
\hline LV33 & $15,10 \pm 1,04 b$ & $4,57 \pm 2,14 \mathrm{c}$ & 3,30 & $* \mathrm{~F}$ \\
\hline LV47 & $14,34 \pm 0,98 \mathrm{c}$ & $4,14 \pm 2,04 \mathrm{e}$ & 3,46 & $* \mathrm{~F}$ \\
\hline LV76 & $15,41 \pm 0,95 \mathrm{a}$ & $4,64 \pm 2,15 d$ & 3,33 & $* \mathrm{~F}$ \\
\hline
\end{tabular}

*C = cilíndrico $* \mathrm{Cc}=$ cilíndrico curto, $* \mathrm{~F}=$ fusiforme $;{ }^{1}$ médias seguidas da mesma letra não diferem estatisticamente, pelo teste de Scott-Knott, a $5 \%$ de probabilidade.

TABELA 3 - Dimensões dos ascósporos $(\mu \mathrm{m})$ dos isolados de Glomerella cingulata f. sp. phaseoli.

\begin{tabular}{lcc}
\hline & Médias das dimensões dos ascósporos & \\
\hline Isolados & Comprimento & Largura \\
\hline LV79 & $21,70 \mathrm{e}^{1}$ & $9,00 \mathrm{c}^{1}$ \\
LV80 & $38,85 \mathrm{a}$ & $10,40 \mathrm{a}$ \\
LV81 & $23,68 \mathrm{~d}$ & $9,90 \mathrm{~b}$ \\
LV82 & $24,43 \mathrm{~d}$ & $6,73 \mathrm{e}$ \\
LV83 & $25,90 \mathrm{c}$ & $8,03 \mathrm{~d}$ \\
LV84 & $35,01 \mathrm{~b}$ & $9,96 \mathrm{~b}$ \\
\hline
\end{tabular}

${ }^{1}$ Médias seguidas da mesma letra não diferem, pelo teste de Scott-Knott (1974), a 5\% de probabilidade.

\section{CONCLUSÕES}

Os isolados de $C$. lindemuthianum, tanto na forma anamórfica como teleomórfica, apresentaram ampla variabilidade para todos os caracteres morfológicos e culturais avaliados.

A forma do conídio de $C$. lindemuthianum pode ser usada como marcador morfológico em estudos genéticos de isolados contrastantes para este caráter.

\section{REFERÊNCIAS BIBLIOGRÁFICAS}

BATISTA, U. G.; CHAVES, G. M. Patogenicidade de culturas monoascospóricas de cruzamento entre raças de Colletotrichum lindemuthianum (Sacc. \& Magn.) Scrib. Fitopatologia Brasileira, Brasília, v. 7 n. 2, p. 285-293, jun. 1982.
BRYSON, R. J. Sexual hibridization and the genetics of pathogenic specificity in Colletotrichum lindemuthianum. 1990. Thesis (Ph.D) - University of Birmingham.

BRYSON, R. J.; CATEN, C. E.; HOLLOMON, D. H.; BAILEY, J. A sexuality and genetics of Colletotrichum In: BAILEY, J. A.; JEGER, M. J. (Ed.). Colletotrichum: biology, pathology and control. Wallingford: C. A. B. International, 1992.

CAMARGO Jr. O. A. Identificação de recombinantes de Glomerella cingulata f. sp. phaseoli por meio de marcadores RAPD. 2004. 60p. Dissertação (Mestrado em Genética e Melhoramento de Plantas) - Universidade Federal de Lavras, Lavras, MG. 
CASTRO, R. A.; MENDES - COSTA, M. C.; SOUZA, E. A.; PALHARES, C. A. Dimorfismo de esporos sexuais de um mutante em Glomerella cingulata f. sp. phaseoli. In: CONGRESSO NACIONAL DE GENÉTICA, 51., 2005, Águas de Lindóia, SP. Resumos... Águas de Lindóia: CDROOM, 2005.

DIAS, M. D. Caracterização morfológica, Bioquímica e Patogênica de isolados de Colletotrichum spp. Em Coffea arabica L. 2002. 64 p. Dissertação (Mestrado em Fitopatologia)-Universidade Federal de Lavras, Lavras, MG.

FEITOSA, M. I.; FEICHTENBERGER, E.; KUDAMATSU, M.; ROSSETTI, V.; LEITE, L. R. Estudos sobre a população de Colletotrichum em Coffea arabica L. no Estado de São Paulo. Arquivo do Instituto Biológico, São Paulo, v. 44, n. 1/2, p. 33-54, jan./jun. 1977.

ISHIKAWA, F. H.; SOUZA, E. A. Caracterização dos isolados de Colletotrichum lindemuthianum (fase assexual) e Glomerella cingulata f. sp phaseoli (fase sexual), agente causal da antracnose no feijoeiro. In: CONGRESSO DE INICIAÇÃO CIENTÍFICA DA UFLACICESAL, 17., 2004, Lavras. Resumos... Lavras: CD-ROOM, 2004.

JUNQUEIRA, N. T. V.; CHAVES, G. M.; ZAMBOLIM, L.; ROMEIRO, R. S.; GASPAROTTO, L. Isolamento, cultivo e esporulação de Microcyclus ulei, agente etiológico do mal das folhas de seringueira. Revista Ceres, Viçosa, v. 31, n. 177, p. 322-331, set./out. 1984.

KIMATI, H.; GALLI, F. Glomerella cingulata (Stonem.) Spauld \& Scherenk. f. sp. phaseoli, fase ascógena do agente causal da antracnose no feijoeiro. Anais da ESALQ, Piracicaba, v. 27, p. 411-437, 1970.

LUCAS, G. B. Genetics of Glomerella IV. Nuclear phenomena in the ascus. American Journal of Botany, Columbus, v. 33, n. 10, p. 802-806, Oct. 1946.
MENDES-COSTA, M. C. Genetics of Glomerella cingulata f. sp. phaseoli 1. Sexual compatibility. Brazilian Journal of Genetics, v. 19, n. 230, p. 350, 1996.

MENDES-COSTA, M. C.; SOUZA, E.A. Genética de Glomerella cingulata (Stonem) Spauld \& Schrenk f. sp. phaseoli : Caracterização genotípica. In: CONGRESSO NACIONAL DE GENÉTICA, 51., 2005, Águas de Lindóia, SP. Resumos... Águas de Lindóia, CD-ROOM, 2005.

MUNSEL ${ }^{\circledR}$ SOIL COLOR CHARTS. Baltimore, Munsell Color Macbeth Division of Kollmorgen Corporation, 1979.

OLIVEIRA, J. A. Efeito do tratamento fungicida em sementes no controle de tombamento de plântulas de pepino (Cucumis sativus L.) e pimentão (Capsicum annum L.). 1991. 111 p. Dissertação (Mestrado em Fitossanidade) Universidade Federal de Lavras, Lavras, MG.

RAVA, A. C.; SARTORATO, A. Antracnose. In: RAVA, A. C.; SARTORATO, A. (Ed). Principais doenças do feijoeiro comum e seu controle. Brasília: EMBRAPA, 1994. p. 17-40.

ROCA, M. M. G.; DAVIDE, C. L; MENDES-COSTA, M. C. Cytogenetics of Colletotrichum lindemuthianum (Glomerella cingulata f. sp. phaseoli). Fitopatologia Brasileira, Brasília, v. 28, n. 4, p. 367-373, jul./ago. 2003.

RODRIGUEZ-GUERRA, R.; RAMIREZ-RUEDA, M. T.; SIMPOSON, J. Towards an understanding of the mechanism which generate variability in Colletotrichum lindemuthianum. In: FUNGAL GENETICS CONFERENCE, 21., 1999, California. Abstracts... California, 1999. p. 49.

SUTTON, B. C. The genus Glomerella and it Anomorph Colletotrichum. In BAYLEY, J. A.; JEGER, M. J. (Ed.). Colletotrichum, biology, pathology and control. Wallingford: C. A. B. international, 1992. p. 1-26. 